\title{
Circuit Analogs for Wave Propagation in Stratified Structures
}

\author{
Daniel Sjöberg \\ Lund University \\ Sweden
}

\section{Introduction}

To simulate or design a reasonably large system, fast and simple models are necessary. To verify the design versus the specifications, more detailed (and costly) calculations can be performed and final adjustments made. In wave propagation problems, circuit analogs provide a powerful, yet simple, means of computing the desired response of the system, such as reflection or transmission coefficients. The reason circuit analog models are good for wave propagation problems, is that they are exact for one-dimensional wave propagation, regardless of whether we consider acoustic or electromagnetic waves.

Typically, wave propagation through homogeneous media is modeled as a transmission line with propagation constant $\beta$ and characteristic impedance $Z$, whereas obstacles such as thin sheets are modeled as lumped elements. If the sheets are lossless, the circuit models contain only reactive elements such as capacitors and inductors.

Modeling complex wave propagation problems with circuit analogs was to a large extent developed in conjunction with the development of radar technology during the Second World War. Many of the results from this very productive era are collected in the Radiation Laboratory Series and related literature, in particular (Collin, 1991; 1992; Marcuvitz, 1951; Schwinger \& Saxon, 1968). Further development has been provided by research on frequency selective structures (Munk, 2000; 2003). In recent years, the circuit analogs have even been used in an inverse fashion: by observing that wave propagation through a material with negative refractive index could be modeled as a transmission line with distributed series capacitance and shunt inductance, i.e., the dual of the standard transmission line, the most successful realization of negative refractive index material is actually made by synthesizing this kind of transmission line using lumped elements (Caloz \& Itoh, 2004; Eleftheriades et al., 2002).

This chapter is organized as follows. In Section 2 we show that propagation of electromagnetic waves in any material, regardless how complicated, boils down to an eigenvalue problem which can be solved analytically for isotropic media, and numerically for arbitrary media. From this eigenvalue problem, the propagation constant and characteristic impedance can be derived, which generates a transmission line model. In Section 3, we show how sheets with or without periodic patterns can be modeled as lumped elements connected by transmission lines representing propagation in the surrounding medium. The lumped elements can be given a firm definition and physical interpretation in the low frequency limit, and in Section 4 we show how these low frequency properties 
provide some useful physical limitations on scattering characteristics. The calculation of circuit analogs in the general case using an optimization approach is treated in Section 5, and examples of the use of circuit analogs in design problems are given in Section 6. Finally, conclusions are given in Section 7.

\section{Wave propagation in stratified structures}

In this section, we show that the description of plane waves propagating through any homogeneous material at any angle of incidence, reduces to a simple eigenvalue problem from which we can compute the propagation constant and transverse wave impedance.

We consider a geometry where the material parameters are constants as functions of $x$ and $y$, but may depend on $z$, which is considered as the main propagation direction. This corresponds to a laminated structure, $z$ being the lamination direction. Our strategy is to eliminate the $x$ and $y$ dependence through a spatial Fourier transform, and then eliminate the field components along the $z$ direction. This is motivated by the fact that the remaining field components, $\boldsymbol{E}_{\mathrm{t}}=E_{x} \hat{\boldsymbol{x}}+E_{y} \hat{\boldsymbol{y}}$ and $\boldsymbol{H}_{\mathrm{t}}=H_{x} \hat{\boldsymbol{x}}+H_{y} \hat{\boldsymbol{y}}$, are continuous across interfaces, and are thus easily matched at boundaries. The resulting equation (24) (or (25) for isotropic media) can be formulated as an algebraic eigenvalue problem by looking for solutions where the only $z$ dependence is through a propagation factor $\mathrm{e}^{-\mathrm{j} \beta z}$. The wave number $\beta$ corresponds to the eigenvalue, and the wave impedance is given by the eigenvectors.

\subsection{Notation}

We consider time harmonic waves using time convention $\mathrm{e}^{\mathrm{j} \omega t}$. The material is described through the mapping from the fields $[E, H]$ to the fields $[D, B]$ :

$$
\left\{\begin{array}{l}
D=\epsilon \cdot E+\xi \cdot H \\
B=\zeta \cdot E+\mu \cdot H
\end{array}\right.
$$

where the dyadics $\epsilon, \xi, \zeta$, and $\mu$ can be represented by $3 \times 3$ matrices. Other mappings for the material are possible, for instance from the fields $[E, B]$ to $[D, H]$. In vacuum the relations are $\boldsymbol{D}=\epsilon_{0} \boldsymbol{E}$ and $\boldsymbol{B}=\mu_{0} \boldsymbol{H}$, where the permittivity and permeability of vacuum are denoted by $\boldsymbol{\epsilon}_{0}=$ $8.854 \cdot 10^{-12} \mathrm{~F} / \mathrm{m}$ and $\mu_{0}=4 \pi \cdot 10^{-7} \mathrm{H} / \mathrm{m}$, respectively. Materials are often classified according to the various symmetries of the material dyadics as in Table 1.

When choosing a particular direction $z$, it is natural to introduce a decomposition as (where the index $t$ represents the $x$ and $y$ components)

$$
E=E_{\mathrm{t}}+\hat{z} E_{z}, \quad \epsilon=\epsilon_{\mathrm{tt}}+\hat{z} \epsilon_{z}+\epsilon_{\mathrm{t}} \hat{z}+\epsilon_{z z} \hat{z} \hat{z}
$$

so that the transverse components of the $\boldsymbol{D}$ and $\boldsymbol{B}$ fields are (vector equations)

\begin{tabular}{l|c|c} 
Type & $\boldsymbol{\epsilon}, \boldsymbol{\mu}$, & $\boldsymbol{\xi}, \zeta$ \\
\hline Isotropic & All $\sim \mathbf{1}$ & Both 0 \\
An-isotropic & Some not $\sim \mathbf{1}$ & Both 0 \\
Bi-isotropic & All $\sim \mathbf{1}$ & Both $\sim \mathbf{1}$ \\
Bi-an-isotropic & \multicolumn{2}{|c}{ All other cases }
\end{tabular}

Table 1. Classification of electromagnetic materials ( $\mathbf{1}$ denotes the unit dyadic). 


$$
\begin{aligned}
& \boldsymbol{D}_{\mathrm{t}}=\boldsymbol{\epsilon}_{\mathrm{tt}} \cdot \boldsymbol{E}_{\mathrm{t}}+\boldsymbol{\epsilon}_{\mathrm{t}} E_{z}+\boldsymbol{\xi}_{\mathrm{tt}} \cdot \boldsymbol{H}_{\mathrm{t}}+\boldsymbol{\xi}_{\mathrm{t}} H_{z} \\
& \boldsymbol{B}_{\mathrm{t}}=\zeta_{\mathrm{tt}} \cdot \boldsymbol{E}_{\mathrm{t}}+\zeta_{\mathrm{t}} E_{z}+\boldsymbol{\mu}_{\mathrm{tt}} \cdot \boldsymbol{H}_{\mathrm{t}}+\boldsymbol{\mu}_{\mathrm{t}} H_{z}
\end{aligned}
$$

and the $z$ components are (scalar equations)

$$
\begin{aligned}
& D_{z}=\boldsymbol{\epsilon}_{z} \cdot \boldsymbol{E}_{\mathrm{t}}+\epsilon_{z z} E_{z}+\boldsymbol{\xi}_{z} \cdot \boldsymbol{H}_{\mathrm{t}}+\xi_{z z} H_{z} \\
& B_{z}=\boldsymbol{\zeta}_{z} \cdot \boldsymbol{E}_{\mathrm{t}}+\zeta_{z z} E_{z}+\boldsymbol{\mu}_{z} \cdot \boldsymbol{H}_{\mathrm{t}}+\mu_{z z} H_{z}
\end{aligned}
$$

Since the material parameters are assumed independent of $x$ and $y$, it makes sense to represent the fields through a Fourier transform in the transverse variables $x$ and $y$ as

$$
\begin{gathered}
\boldsymbol{E}(\boldsymbol{r})=\frac{1}{(2 \pi)^{2}} \int_{-\infty}^{\infty} \int_{-\infty} \boldsymbol{E}\left(z, \boldsymbol{k}_{\mathrm{t}}\right) \mathrm{e}^{-\mathrm{j}\left(k_{x} x+k_{y} y\right)} d k_{x} d k_{y} \\
\boldsymbol{E}\left(z, \boldsymbol{k}_{\mathrm{t}}\right)=\int_{-\infty}^{\infty} \int_{-\infty} \boldsymbol{E}(\boldsymbol{r}) \mathrm{e}^{\mathrm{j}\left(k_{x} x+k_{y} y\right)} d x d y
\end{gathered}
$$

where the transverse wave vector is $k_{\mathrm{t}}=k_{x} \hat{\boldsymbol{x}}+k_{y} \hat{\boldsymbol{y}}$. The action of the curl operator on the Fourier amplitude is shown by

$$
\begin{aligned}
\nabla \times \boldsymbol{E}(\boldsymbol{r}) & =\frac{1}{(2 \pi)^{2}} \int_{-\infty}^{\infty} \int_{-\infty} \times\left[\boldsymbol{E}\left(z, \boldsymbol{k}_{\mathrm{t}}\right) \mathrm{e}^{-\mathrm{j}\left(k_{x} x+k_{y} y\right)}\right] d k_{x} d k_{y} \\
& =\frac{1}{(2 \pi)^{2}} \int_{-\infty}^{\infty} \int^{-\mathrm{j}\left(k_{x} x+k_{y} y\right)}\left(-\mathrm{j} \boldsymbol{k}_{\mathrm{t}}+\hat{z} \frac{\partial}{\partial z}\right) \times \boldsymbol{E}\left(z, \boldsymbol{k}_{\mathrm{t}}\right) d k_{x} d k_{y}
\end{aligned}
$$

We then obtain the decomposition

$$
\begin{aligned}
\left(-\mathrm{j} \boldsymbol{k}_{\mathrm{t}}+\hat{z} \frac{\partial}{\partial z}\right) \times \boldsymbol{E}\left(z, \boldsymbol{k}_{\mathrm{t}}\right) & =-\mathrm{j} \boldsymbol{k}_{\mathrm{t}} \times \boldsymbol{E}\left(z, \boldsymbol{k}_{\mathrm{t}}\right)+\frac{\partial}{\partial z} \hat{\boldsymbol{z}} \times \boldsymbol{E}\left(z, \boldsymbol{k}_{\mathrm{t}}\right) \\
& =-\underbrace{\mathrm{j} \boldsymbol{k}_{\mathrm{t}} \times \boldsymbol{E}_{\mathrm{t}}\left(z, \boldsymbol{k}_{\mathrm{t}}\right)}_{\text {parallel to } \hat{z}}-\underbrace{j \boldsymbol{k}_{\mathrm{t}} \times \hat{\boldsymbol{z}} E_{\mathrm{z}}\left(z, \boldsymbol{k}_{\mathrm{t}}\right)}_{\text {orthogonal to } \hat{z}}+\underbrace{\frac{\partial}{\partial z} \hat{z} \times \boldsymbol{E}_{\mathrm{t}}\left(z, \boldsymbol{k}_{\mathrm{t}}\right)}_{\text {orthogonal to } \hat{z}}
\end{aligned}
$$

The result for the curl of the magnetic field is exactly the same.

\subsection{Application to Maxwell's equations}

We now apply the above decompositions with respect to $z$ to Maxwell's equations. These are

$$
\begin{aligned}
& \nabla \times H(r)=j \omega D(r) \\
& \nabla \times E(r)=-j \omega B(r)
\end{aligned}
$$

When considering the Fourier amplitudes of the electromagnetic fields and using the constitutive relations this turns into (in the following we suppress the arguments $z$ and $k_{\mathrm{t}}$ of the fields for brevity) 


$$
\begin{aligned}
& \left(-\mathrm{j} \boldsymbol{k}_{\mathrm{t}}+\hat{z} \frac{\partial}{\partial z}\right) \times \boldsymbol{H}=\mathrm{j} \omega(\boldsymbol{\epsilon} \cdot \boldsymbol{E}+\boldsymbol{\xi} \cdot \boldsymbol{H}) \\
& \left(-\mathrm{j} \boldsymbol{k}_{\mathrm{t}}+\hat{z} \frac{\partial}{\partial z}\right) \times \boldsymbol{E}=-\mathrm{j} \omega(\zeta \cdot \boldsymbol{E}+\boldsymbol{\mu} \cdot \boldsymbol{H})
\end{aligned}
$$

Another way to write this is by using dyadics (identifying (13) as the first row and (14) as the second row, and writing 1 for the unit dyadic)

$$
\frac{\partial}{\partial z}\left(\begin{array}{cc}
\mathbf{0} & -\hat{z} \times \mathbf{1} \\
\hat{z} \times \mathbf{1} & \mathbf{0}
\end{array}\right) \cdot\left(\begin{array}{c}
E \\
H
\end{array}\right)=\left(\begin{array}{cc}
0 & -\mathrm{j} k_{\mathrm{t}} \times \mathbf{1} \\
\mathrm{j} \boldsymbol{k}_{\mathrm{t}} \times \mathbf{1} & \mathbf{0}
\end{array}\right) \cdot\left(\begin{array}{c}
E \\
H
\end{array}\right)-\mathrm{j} \omega\left(\begin{array}{cc}
\epsilon & \xi \\
\zeta & \mu
\end{array}\right) \cdot\left(\begin{array}{c}
E \\
H
\end{array}\right)
$$

The left hand side is orthogonal to $\hat{z}$, and the equations for the $z$ components are then (using that the cross product $k_{\mathrm{t}} \times E_{\mathrm{t}}$ is necessarily in the $z$ direction since both vectors are in the $x y$-plane, with the scalar value $\left.\hat{z} \cdot\left(k_{\mathrm{t}} \times E_{\mathrm{t}}\right)=\left(\hat{z} \times k_{\mathrm{t}}\right) \cdot E_{\mathrm{t}}\right)$

$$
\left(\begin{array}{l}
0 \\
0
\end{array}\right)=\left(\begin{array}{cc}
\mathbf{0} & -\mathrm{j} \hat{z} \times \boldsymbol{k}_{\mathrm{t}} \\
\mathrm{j} \hat{z} \times \boldsymbol{k}_{\mathrm{t}} & \mathbf{0}
\end{array}\right) \cdot\left(\begin{array}{c}
\boldsymbol{E}_{\mathrm{t}} \\
\boldsymbol{H}_{\mathrm{t}}
\end{array}\right)-\mathrm{j} \omega\left(\begin{array}{cc}
\boldsymbol{\epsilon}_{z} & \boldsymbol{\xi}_{z} \\
\boldsymbol{\zeta}_{z} & \boldsymbol{\mu}_{z}
\end{array}\right) \cdot\left(\begin{array}{c}
\boldsymbol{E}_{\mathrm{t}} \\
\boldsymbol{H}_{\mathrm{t}}
\end{array}\right)-\mathrm{j} \omega\left(\begin{array}{cc}
\epsilon_{z z} & \boldsymbol{\xi}_{z z} \\
\zeta_{z z} & \mu_{z z}
\end{array}\right)\left(\begin{array}{c}
E_{z} \\
H_{z}
\end{array}\right)
$$

from which we solve for the $z$ components of the fields:

$$
\left(\begin{array}{c}
E_{z} \\
H_{z}
\end{array}\right)=\left(\begin{array}{cc}
\epsilon_{z z} & \xi_{z z} \\
\zeta_{z z} & \mu_{z z}
\end{array}\right)^{-1}\left[\left(\begin{array}{cc}
\mathbf{0} & -\omega^{-1} \hat{\boldsymbol{z}} \times \boldsymbol{k}_{\mathrm{t}} \\
\omega^{-1} \hat{\boldsymbol{z}} \times \boldsymbol{k}_{\mathrm{t}} & \mathbf{0}
\end{array}\right)-\left(\begin{array}{cc}
\boldsymbol{\epsilon}_{z} & \boldsymbol{\xi}_{z} \\
\boldsymbol{\zeta}_{z} & \boldsymbol{\mu}_{z}
\end{array}\right)\right] \cdot\left(\begin{array}{c}
\boldsymbol{E}_{\mathrm{t}} \\
\boldsymbol{H}_{\mathrm{t}}
\end{array}\right)
$$

The transverse part of (15) is

$$
\begin{gathered}
\frac{\partial}{\partial z}\left(\begin{array}{cc}
\mathbf{0} & -\hat{\boldsymbol{z}} \times \mathbf{1} \\
\hat{\boldsymbol{z}} \times \mathbf{1} & \mathbf{0}
\end{array}\right) \cdot\left(\begin{array}{l}
\boldsymbol{E}_{\mathrm{t}} \\
\boldsymbol{H}_{\mathrm{t}}
\end{array}\right)=\left(\begin{array}{cc}
\mathbf{0} & -\mathrm{j} \boldsymbol{k}_{\mathrm{t}} \times \hat{\boldsymbol{z}} \\
\mathrm{j} \boldsymbol{k}_{\mathrm{t}} \times \hat{\boldsymbol{z}} & \mathbf{0}
\end{array}\right)\left(\begin{array}{c}
E_{z} \\
H_{z}
\end{array}\right) \\
-\mathrm{j} \omega\left(\begin{array}{cc}
\boldsymbol{\epsilon}_{\mathrm{tt}} & \boldsymbol{\xi}_{\mathrm{tt}} \\
\boldsymbol{\zeta}_{\mathrm{tt}} & \boldsymbol{\mu}_{\mathrm{tt}}
\end{array}\right) \cdot\left(\begin{array}{c}
\boldsymbol{E}_{\mathrm{t}} \\
\boldsymbol{H}_{\mathrm{t}}
\end{array}\right)-\mathrm{j} \omega\left(\begin{array}{ll}
\boldsymbol{\epsilon}_{\mathrm{t}} & \boldsymbol{\xi}_{\mathrm{t}} \\
\boldsymbol{\zeta}_{\mathrm{t}} & \boldsymbol{\mu}_{\mathrm{t}}
\end{array}\right)\left(\begin{array}{c}
E_{z} \\
H_{z}
\end{array}\right) \\
=-\mathrm{j} \omega\left(\begin{array}{ll}
\boldsymbol{\epsilon}_{\mathrm{tt}} & \boldsymbol{\xi}_{\mathrm{tt}} \\
\boldsymbol{\zeta}_{\mathrm{tt}} & \boldsymbol{\mu}_{\mathrm{tt}}
\end{array}\right) \cdot\left(\begin{array}{l}
\boldsymbol{E}_{\mathrm{t}} \\
\boldsymbol{H}_{\mathrm{t}}
\end{array}\right)+\mathrm{j} \omega\left[\left(\begin{array}{cc}
0 & -\omega^{-1} \boldsymbol{k}_{\mathrm{t}} \times \hat{z} \\
\omega^{-1} \boldsymbol{k}_{\mathrm{t}} \times \hat{z} & 0
\end{array}\right)-\left(\begin{array}{cc}
\boldsymbol{\epsilon}_{\mathrm{t}} & \boldsymbol{\xi}_{\mathrm{t}} \\
\boldsymbol{\zeta}_{\mathrm{t}} & \boldsymbol{\mu}_{\mathrm{tt}}
\end{array}\right)\right]\left(\begin{array}{c}
E_{z} \\
H_{z}
\end{array}\right)
\end{gathered}
$$

Inserting the expressions for the $z$ components of the fields implies

$$
\frac{\partial}{\partial z}\left(\begin{array}{cc}
\mathbf{0} & -\hat{\boldsymbol{z}} \times \mathbf{1} \\
\hat{\boldsymbol{z}} \times \mathbf{1} & \mathbf{0}
\end{array}\right) \cdot\left(\begin{array}{c}
\boldsymbol{E}_{\mathrm{t}} \\
\boldsymbol{H}_{\mathrm{t}}
\end{array}\right)=-\mathrm{j} \omega\left(\begin{array}{cc}
\boldsymbol{\epsilon}_{\mathrm{tt}} & \boldsymbol{\xi}_{\mathrm{tt}} \\
\boldsymbol{\zeta}_{\mathrm{tt}} & \boldsymbol{\mu}_{\mathrm{tt}}
\end{array}\right) \cdot\left(\begin{array}{c}
\boldsymbol{E}_{\mathrm{t}} \\
\boldsymbol{H}_{\mathrm{t}}
\end{array}\right)+\mathrm{j} \omega \mathbf{A} \cdot\left(\begin{array}{c}
\boldsymbol{E}_{\mathrm{t}} \\
\boldsymbol{H}_{\mathrm{t}}
\end{array}\right)
$$

where $\mathbf{A}$ is the dyadic product ${ }^{1}$

1 A dyadic product between two vectors $\boldsymbol{a} \boldsymbol{b}$ is defined by its action on an arbitrary vector $\boldsymbol{c}$ as $(\boldsymbol{a} \boldsymbol{b}) \cdot \boldsymbol{c}=\boldsymbol{a}(\boldsymbol{b} \cdot \boldsymbol{c})$, i.e., a vector parallel to $\boldsymbol{a}$ with amplitude $|\boldsymbol{a}||\boldsymbol{b} \cdot \boldsymbol{c}|$. Thus, dyadic multiplication does not commute unless $\boldsymbol{a}$ is parallel to $\boldsymbol{b}$. 


$$
\mathbf{A}=\left[\left(\begin{array}{cc}
\mathbf{0} & -\omega^{-1} \boldsymbol{k}_{\mathrm{t}} \times \hat{\boldsymbol{z}} \\
\omega^{-1} \boldsymbol{k}_{\mathrm{t}} \times \hat{z} & \mathbf{0}
\end{array}\right)-\left(\begin{array}{cc}
\boldsymbol{\epsilon}_{\mathrm{t}} & \boldsymbol{\xi}_{\mathrm{t}} \\
\boldsymbol{\zeta}_{\mathrm{t}} & \boldsymbol{\mu}_{\mathrm{t}}
\end{array}\right)\right]\left(\begin{array}{cc}
\epsilon_{z z} & \boldsymbol{\xi}_{z z} \\
\zeta_{z z} & \mu_{z z}
\end{array}\right)^{-1}\left[\left(\begin{array}{cc}
\mathbf{0} & -\omega^{-1} \hat{\boldsymbol{z}} \times \boldsymbol{k}_{\mathrm{t}} \\
\omega^{-1} \hat{\boldsymbol{z}} \times \boldsymbol{k}_{\mathrm{t}} & \mathbf{0}
\end{array}\right)-\left(\begin{array}{cc}
\boldsymbol{\epsilon}_{z} & \boldsymbol{\xi}_{z} \\
\boldsymbol{\zeta}_{z} & \boldsymbol{\mu}_{z}
\end{array}\right)\right]
$$

For an isotropic material, where $\boldsymbol{\epsilon}=\epsilon \mathbf{1}, \boldsymbol{\mu}=\mu \mathbf{1}, \boldsymbol{\xi}=\boldsymbol{\zeta}=\mathbf{0}$, this is (writing $\boldsymbol{a}=k_{0}^{-1} \boldsymbol{k}_{\mathrm{t}} \times \hat{z}=$ $-k_{0}^{-1} \hat{z} \times k_{\mathrm{t}}$ where $k_{0}=\omega / \mathrm{c}_{0}$ is the wave number in vacuum)

$$
\mathbf{A}=\left(\begin{array}{cc}
\mathbf{0} & -\mathrm{c}_{0}^{-1} \boldsymbol{a} \\
\mathrm{c}_{0}^{-1} \boldsymbol{a} & \mathbf{0}
\end{array}\right)\left(\begin{array}{cc}
\epsilon^{-1} & 0 \\
0 & \mu^{-1}
\end{array}\right)\left(\begin{array}{cc}
\mathbf{0} & \mathrm{c}_{0}^{-1} \boldsymbol{a} \\
-\mathrm{c}_{0}{ }^{-1} \boldsymbol{a} & \mathbf{0}
\end{array}\right)=\frac{1}{\mathrm{c}_{0}{ }^{2}}\left(\begin{array}{cc}
\mu^{-1} \boldsymbol{a} \boldsymbol{a} & \mathbf{0} \\
\mathbf{0} & \epsilon^{-1} \boldsymbol{a} \boldsymbol{a}
\end{array}\right)
$$

Since $-\hat{z} \times\left(\hat{z} \times E_{\mathrm{t}}\right)=E_{\mathrm{t}}$ for all transverse fields $\boldsymbol{E}_{\mathrm{t}}$, we can write (19) as

$$
\frac{\partial}{\partial z}\left(\begin{array}{c}
\boldsymbol{E}_{\mathrm{t}} \\
-\hat{z} \times \boldsymbol{H}_{\mathrm{t}}
\end{array}\right)=\left(\begin{array}{cc}
\mathbf{0} & -\hat{z} \times \mathbf{1} \\
\mathbf{1} & \mathbf{0}
\end{array}\right) \cdot\left[-\mathrm{j} \omega\left(\begin{array}{cc}
\boldsymbol{\epsilon}_{\mathrm{tt}} & \boldsymbol{\xi}_{\mathrm{tt}} \\
\boldsymbol{\zeta}_{\mathrm{tt}} & \boldsymbol{\mu}_{\mathrm{tt}}
\end{array}\right)+\mathrm{j} \omega \mathbf{A}\right] \cdot\left(\begin{array}{cc}
\mathbf{1} & \mathbf{0} \\
\mathbf{0} & \hat{z} \times \mathbf{1}
\end{array}\right) \cdot\left(\begin{array}{c}
\boldsymbol{E}_{\mathrm{t}} \\
-\hat{\boldsymbol{z}} \times \boldsymbol{H}_{\mathrm{t}}
\end{array}\right)
$$

By keeping the vector product with $\hat{z}$ in the magnetic field, the vectors $E_{\mathrm{t}}$ and $-\hat{z} \times H_{\mathrm{t}}$ will be parallel to each other in isotropic media. Identifying the transverse electric and magnetic fields as vector voltage and vector current, i.e.,

we find

$$
E_{\mathrm{t}}=V \text { and }-\hat{z} \times H_{\mathrm{t}}=\boldsymbol{I}
$$

$$
\frac{\partial}{\partial z}\left(\begin{array}{l}
\boldsymbol{I} \\
\boldsymbol{I}
\end{array}\right)=\left(\begin{array}{cc}
\mathbf{0} & -\hat{\boldsymbol{z}} \times \mathbf{1} \\
\mathbf{1} & \mathbf{0}
\end{array}\right) \cdot\left[-\mathrm{j} \omega\left(\begin{array}{cc}
\boldsymbol{\epsilon}_{\mathrm{tt}} & \boldsymbol{\xi}_{\mathrm{tt}} \\
\boldsymbol{\zeta}_{\mathrm{tt}} & \boldsymbol{\mu}_{\mathrm{tt}}
\end{array}\right)+\mathrm{j} \omega \mathbf{A}\right] \cdot\left(\begin{array}{cc}
\mathbf{1} & \mathbf{0} \\
\mathbf{0} & \hat{z} \times \mathbf{1}
\end{array}\right) \cdot\left(\begin{array}{l}
\boldsymbol{V} \\
\boldsymbol{I}
\end{array}\right)
$$

In particular, for an isotropic material this simplifies to

$$
\frac{\partial}{\partial z}\left(\begin{array}{l}
\boldsymbol{I} \\
\boldsymbol{I}
\end{array}\right)=\left[-\mathrm{j} \omega\left(\begin{array}{cc}
\mathbf{0} & \mu \mathbf{1} \\
\epsilon \mathbf{1} & \mathbf{0}
\end{array}\right)+\frac{\mathrm{j} \omega}{\mathrm{c}_{0}^{2}}\left(\begin{array}{cc}
0 & \epsilon^{-1} \boldsymbol{b} \boldsymbol{b} \\
\mu^{-1} \boldsymbol{a} \boldsymbol{a} & 0
\end{array}\right)\right] \cdot\left(\begin{array}{l}
\boldsymbol{I} \\
\boldsymbol{I}
\end{array}\right)
$$

where the unitless vector $\boldsymbol{a}=k_{0}^{-1} \boldsymbol{k}_{\mathrm{t}} \times \hat{z}$ defines the direction of the TE polarized transverse electric field (electric field transverse to the plane of incidence), and the unitless vector $\boldsymbol{b}=$ $\hat{z} \times a=k_{0}^{-1} \boldsymbol{k}_{\mathrm{t}}$ defines the direction of the TM polarized transverse electric field (electric field in the plane of incidence). The amplitude of both vectors is $|\boldsymbol{a}|=|\boldsymbol{b}|=\left|\boldsymbol{k}_{\mathrm{t}}\right| / k_{0}=\sin \theta$, where $\theta$ is the angle of incidence in vacuum.

Equation (24) is recognized as a linear dynamical system for the transverse field components. If the material parameters are constant with respect to $z$, the solution of (24) can be written using the exponential matrix as (where $V_{1}=V\left(z_{1}\right)$ and $V_{2}=V\left(z_{2}\right)$ etc)

$$
\left(\begin{array}{l}
\boldsymbol{V}_{1} \\
\boldsymbol{I}_{1}
\end{array}\right)=\exp \left\{\left(z_{1}-z_{2}\right)\left(\begin{array}{cc}
\mathbf{0} & -\hat{\boldsymbol{z}} \times \mathbf{1} \\
\mathbf{1} & \mathbf{0}
\end{array}\right) \cdot\left[-\mathrm{j} \omega\left(\begin{array}{cc}
\boldsymbol{\epsilon}_{\mathrm{tt}} & \boldsymbol{\xi}_{\mathrm{tt}} \\
\boldsymbol{\zeta}_{\mathrm{tt}} & \boldsymbol{\mu}_{\mathrm{tt}}
\end{array}\right)+\mathrm{j} \omega \mathbf{A}\right] \cdot\left(\begin{array}{cc}
\mathbf{1} & \mathbf{0} \\
\mathbf{0} & \hat{\boldsymbol{z}} \times \mathbf{1}
\end{array}\right)\right\} \cdot\left(\begin{array}{l}
\boldsymbol{V}_{2} \\
\boldsymbol{I}_{2}
\end{array}\right)=\mathbf{P}\left(z_{1}, z_{2}\right) \cdot\left(\begin{array}{l}
\boldsymbol{V}_{2} \\
\boldsymbol{I}_{2}
\end{array}\right)
$$

This formal solution reveals an important structure, which generalizes to inhomogeneous media where the material parameters may depend on $z$ : the transverse fields at $z=z_{1}$ can be written as a dyadic $\mathbf{P}$ operating on the fields at $z=z_{2}$, where $z_{1}$ and $z_{2}$ are arbitrary (although the dyadic of course depends on $z_{1}$ and $z_{2}$ ). This dyadic is called a propagator, and its 
existence is guaranteed by the linearity of the problem. We write the explicit form of this dyadic for isotropic media in (34), but first we must define a few properties.

\subsection{Eigenvalue problem in infinite media}

If the wave is propagating in a medium which is infinite in the $z$ direction, it is natural to search for solutions on the form

$$
\left(\begin{array}{c}
\boldsymbol{V}(z) \\
\boldsymbol{I}(z)
\end{array}\right)=\left(\begin{array}{c}
\boldsymbol{V}_{0} \\
\boldsymbol{I}_{0}
\end{array}\right) \mathrm{e}^{-\mathrm{j} \beta z}
$$

This implies $\frac{\partial}{\partial z}[V(z), I(z)]=-\mathrm{j} \beta\left[V_{0}, I_{0}\right] \mathrm{e}^{-\mathrm{j} \beta z}$ which makes (24) turn into an algebraic eigenvalue problem (after dividing by $-\mathrm{j} \omega$ and the exponential factor $\mathrm{e}^{-\mathrm{j} \beta z}$ )

$$
\frac{\beta}{\omega}\left(\begin{array}{c}
\boldsymbol{V}_{0} \\
\boldsymbol{I}_{0}
\end{array}\right)=\left(\begin{array}{cc}
\mathbf{0} & -\hat{z} \times \mathbf{1} \\
\mathbf{1} & \mathbf{0}
\end{array}\right) \cdot\left[\left(\begin{array}{cc}
\boldsymbol{\epsilon}_{\mathrm{tt}} & \boldsymbol{\xi}_{\mathrm{tt}} \\
\boldsymbol{\zeta}_{\mathrm{tt}} & \boldsymbol{\mu}_{\mathrm{tt}}
\end{array}\right)-\mathbf{A}\right] \cdot\left(\begin{array}{cc}
\mathbf{1} & \mathbf{0} \\
\mathbf{0} & \hat{z} \times \mathbf{1}
\end{array}\right) \cdot\left(\begin{array}{c}
\boldsymbol{V}_{0} \\
\boldsymbol{I}_{0}
\end{array}\right)
$$

Thus, the propagation constant $\beta$ can be found from the eigenvalue problem (28), which can easily be solved numerically once the material model is specified along with the transverse wave vector $\boldsymbol{k}_{\mathrm{t}}$ (which occurs only in $\mathbf{A}$ ).

In addition, the field amplitudes $\left[\boldsymbol{V}_{0}, \boldsymbol{I}_{0}\right]$ are the eigenvectors of the same dyadic and can be determined up to a multiplicative constant. Independent of the normalization, the eigenvectors always provide a mapping between the transverse components of the electric and magnetic fields, i.e.,

$$
V_{0}=\mathbf{Z} \cdot \mathbf{I}_{0}
$$

where the dyadic $\mathbf{Z}$ is the transverse wave impedance of the wave. For isotropic media corresponding to (25), we have

$$
\frac{\beta}{\omega}\left(\begin{array}{c}
\boldsymbol{V}_{0} \\
\boldsymbol{I}_{0}
\end{array}\right)=\left(\begin{array}{cc}
0 & \mu-\mathrm{c}_{0}^{-2} \epsilon^{-1} \boldsymbol{b} \boldsymbol{b} \\
\epsilon-\mathrm{c}_{0}^{-2} \mu^{-1} \boldsymbol{a} \boldsymbol{a} & 0
\end{array}\right) \cdot\left(\begin{array}{l}
\boldsymbol{V}_{0} \\
\boldsymbol{I}_{0}
\end{array}\right)
$$

This implies

$$
\left(\frac{\beta}{\omega}\right)^{2} V_{0}=\epsilon \mu\left(1-\frac{\mathrm{c}_{0}^{-2}}{\epsilon \mu} a \boldsymbol{a}\right) \cdot\left(\mathbf{1}-\frac{\mathrm{c}_{0}^{-2}}{\epsilon \mu} \boldsymbol{b} \boldsymbol{b}\right) \cdot \boldsymbol{V}_{0}=\epsilon \mu\left(\mathbf{1}-\frac{\mathrm{c}_{0}^{-2}}{\epsilon \mu}(\boldsymbol{a} \boldsymbol{a}+\boldsymbol{b} \boldsymbol{b})\right) \cdot \boldsymbol{V}_{0}=\left(\epsilon \mu-\mathrm{c}_{0}^{-2} \sin ^{2} \theta\right) \boldsymbol{V}_{0}
$$

where we used $\boldsymbol{a} \cdot \boldsymbol{b}=0$ and $|\boldsymbol{a}|=|\boldsymbol{b}|=\sin \theta$. The final result for the wave number is then

$$
\beta=\omega \sqrt{\varepsilon \mu-\mathrm{c}_{0}^{-2} \sin ^{2} \theta}
$$

and the wave impedance, defined by the relation $V_{0}=\mathbf{Z} \cdot I_{0}$, is

$$
\mathrm{Z}=\frac{\omega}{\beta} \mu\left(\mathbf{1}-\frac{\mathrm{c}_{0}^{-2}}{\varepsilon \mu} \boldsymbol{b} \boldsymbol{b}\right)=\frac{\omega \mu}{\beta} \frac{\boldsymbol{a} \boldsymbol{a}}{|\boldsymbol{a}|^{2}}+\frac{\beta}{\omega \varepsilon} \frac{\boldsymbol{b} \boldsymbol{b}}{|\boldsymbol{b}|^{2}}
$$


In vacuum, we have $\omega \mu / \beta=\eta_{0} / \cos \theta$ and $\beta /(\omega \epsilon)=\eta_{0} \cos \theta$, where $\eta_{0}=\sqrt{\mu_{0} / \epsilon_{0}}$ is the intrinsic wave impedance of vacuum. Finally, the propagator dyadic for a slab of length $\ell$ of isotropic media is

$$
\mathbf{P}=\left(\begin{array}{cc}
\cos (\beta \ell) \mathbf{1} & \mathrm{j} \sin (\beta \ell) \mathbf{Z} \\
\mathrm{j} \sin (\beta \ell) \mathbf{Z}^{-1} & \cos (\beta \ell) \mathbf{1}
\end{array}\right)
$$

In microwave theory, this is recognized as the $A B C D$-matrix of a transmission line with propagation constant $\beta$ and characteristic impedance $\mathbf{Z}$ (Pozar, 2005, p. 185). Note however that we have generalized it to include both TE and TM polarization, through the dyadic character of $\mathbf{Z}$. The important thing about the propagator dyadic is that since tangential electric and magnetic fields are continuous, we can find the total propagator dyadic for a layered structure by cascading:

$$
\mathbf{P}_{\text {tot }}=\mathbf{P}_{1} \cdot \mathbf{P}_{2} \cdots \mathbf{P}_{N}=\left(\begin{array}{cc}
\mathbf{A} & \mathbf{B} \\
\mathbf{C} & \mathbf{D}
\end{array}\right) \text {, where }\left(\begin{array}{l}
\boldsymbol{V}_{1} \\
\boldsymbol{I}_{1}
\end{array}\right)=\mathbf{P}_{\text {tot }} \cdot\left(\begin{array}{l}
\boldsymbol{V}_{2} \\
\boldsymbol{I}_{2}
\end{array}\right)
$$

The dyadic $\mathbf{P}_{\text {tot }}$ maps the total fields from one side of the layered structure to the other. Outside the structure, the total fields can be expressed in terms of the incident field amplitude $V_{\text {inc }}$ using reflection and transmission dyadics $\mathbf{r}$ and $\mathbf{t}$ as (where we assume the same medium on both sides, with the characteristic impedance $\mathbf{Z}_{0}$, and use the fact that waves propagating along the positive $z$ direction satisfy $V_{+}=Z_{0} \cdot I_{+}$, whereas waves propagating in the negative $z$ direction satisfy $\left.V_{-}=-Z_{0} \cdot I_{-}\right)$

$$
\left(\begin{array}{c}
\boldsymbol{V}_{1} \\
\boldsymbol{I}_{1}
\end{array}\right)=\left(\begin{array}{c}
(\mathbf{1}+\mathbf{r}) \cdot \boldsymbol{V}_{\mathrm{inc}} \\
\mathbf{Z}_{0}^{-1} \cdot(\mathbf{1}-\mathbf{r}) \cdot \boldsymbol{V}_{\mathrm{inc}}
\end{array}\right) \text { and }\left(\begin{array}{c}
\boldsymbol{V}_{2} \\
\boldsymbol{I}_{2}
\end{array}\right)=\left(\begin{array}{c}
\mathbf{t} \cdot \boldsymbol{V}_{\mathrm{inc}} \\
\mathbf{Z}_{0}^{-1} \cdot \mathbf{t} \cdot \boldsymbol{V}_{\mathrm{inc}}
\end{array}\right)
$$

Solving for the reflection and transmission dyadics imply

$$
\begin{gathered}
\mathbf{r}=\left(\mathbf{A}+\mathbf{B} \cdot \mathbf{Z}_{0}^{-1}-\mathbf{Z}_{0} \cdot \mathbf{C}-\mathbf{Z}_{0} \cdot \mathbf{D} \cdot \mathbf{Z}_{0}^{-1}\right) \cdot\left(\mathbf{A}+\mathbf{B} \cdot \mathbf{Z}_{0}^{-1}+\mathbf{Z}_{0} \cdot \mathbf{C}+\mathbf{Z}_{0} \cdot \mathbf{D} \cdot \mathbf{Z}_{0}^{-1}\right)^{-1} \\
\mathbf{t}=2\left(\mathbf{A}+\mathbf{B} \cdot \mathbf{Z}_{0}^{-1}+\mathbf{Z}_{0} \cdot \mathbf{C}+\mathbf{Z}_{0} \cdot \mathbf{D} \cdot \mathbf{Z}_{0}^{-1}\right)^{-1}
\end{gathered}
$$

Thus, the concept of propagator dyadics enables a straight-forward analysis of layered structures, although the final results in terms of reflection and transmission coefficients may be complicated. In addition, thin sheets which are inhomogeneous in the $x y$-plane can also be modeled with corresponding propagator dyadics. This is explored in the next sections.

\section{Lumped element models of scatterers}

In real applications, relatively thick homogeneous slabs are often interlaced with thinner sheets, which may also be inhomogeneous in the transverse plane. Such scatterers can be modeled as lumped elements, the simplest of which corresponds to homogeneous, thin sheets. We are thus led to study the limit of the ABCD-matrix for a slab when its thickness $\ell$ becomes small. Denote the thickness of the sheet by $t$. Considering the factors in the propagator dyadic (34) and keeping factors to first order in $\beta t$, we find

$$
\cos (\beta t) \rightarrow 1 \text { and } \sin (\beta t) \rightarrow \beta t
$$


Thus, to first order in $\beta t$ the ABCD-matrix is (using $\mathrm{Z}=\frac{\omega \mu}{\beta} \frac{\boldsymbol{a} \boldsymbol{a}}{|\boldsymbol{a}|^{2}}+\frac{\beta}{\omega \epsilon} \frac{\boldsymbol{b} \boldsymbol{b}}{|\boldsymbol{b}|^{2}}$ )

$$
\left(\begin{array}{cc}
\cos (\beta t) \mathbf{1} & \mathrm{j} \sin (\beta t) \mathbf{Z} \\
\mathrm{j} \sin (\beta t) \mathbf{Z}^{-1} & \cos (\beta t) \mathbf{1}
\end{array}\right) \rightarrow\left(\begin{array}{cc}
\mathbf{1} & \mathrm{j} \omega \mu t\left(\frac{\boldsymbol{a} \boldsymbol{a}}{|\boldsymbol{a}|^{2}}+\frac{\beta^{2}}{\omega^{2} \epsilon \mu} \frac{\boldsymbol{b} \boldsymbol{b}}{|\boldsymbol{b}|^{2}}\right) \\
\mathrm{j} \omega \varepsilon t\left(\frac{\beta^{2}}{\omega^{2} \epsilon \mu} \frac{\boldsymbol{a} \boldsymbol{a}}{|\boldsymbol{a}|^{2}}+\frac{\boldsymbol{b} \boldsymbol{b}}{|\boldsymbol{b}|^{2}}\right) & \mathbf{1}
\end{array}\right)
$$

In order to treat the sheet as a lumped element, the reference planes $T$ and $T^{\prime}$ in Figure 1 should coincide. This corresponds to back propagating the fields at $T^{\prime}$ by multiplying the dyadic above by the inverse of the corresponding dyadic for the background medium (denoted by index 0 ), or to first order in $\beta t$, subtracting the corresponding phase change in the off-diagonal elements. For instance, the upper right element should be replaced by (using $\beta^{2}=\epsilon \mu-\mathrm{c}_{0}^{-2} \sin ^{2} \theta$ and $\mathrm{c}_{0}^{-2}=\epsilon_{0} \mu_{0}$ )

$$
\begin{aligned}
& \mathrm{j} \omega \mu t\left[\frac{\boldsymbol{a} \boldsymbol{a}}{|\boldsymbol{a}|^{2}}+\left(1-\frac{\mathrm{c}_{0}^{-2}}{\epsilon \mu} \sin ^{2} \theta\right) \frac{\boldsymbol{b} \boldsymbol{b}}{|\boldsymbol{b}|^{2}}\right]-\mathrm{j} \omega \mu_{0} t\left[\frac{\boldsymbol{a} \boldsymbol{a}}{|\boldsymbol{a}|^{2}}+\left(1-\frac{\mathrm{c}_{0}^{-2}}{\epsilon_{0} \mu_{0}} \sin ^{2} \theta\right) \frac{\boldsymbol{b} \boldsymbol{b}}{|\boldsymbol{b}|^{2}}\right] \\
& =\mathrm{j} \omega\left(\mu-\mu_{0}\right) t\left(\frac{\boldsymbol{a} \boldsymbol{a}}{|\boldsymbol{a}|^{2}}+\frac{\boldsymbol{b} \boldsymbol{b}}{|\boldsymbol{b}|^{2}}\right)+\left(-\mathrm{j} \omega \mu t \frac{\mathrm{c}_{0}^{-2}}{\epsilon \mu} \sin ^{2} \theta+\mathrm{j} \omega \mu_{0} t \sin ^{2} \theta\right) \frac{\boldsymbol{b} \boldsymbol{b}}{|\boldsymbol{b}|^{2}} \\
& =\mathrm{j} \omega\left(\mu-\mu_{0}\right) t \mathbf{1}+\mathrm{j} \omega \mu_{0} t_{\sin ^{2}} \theta\left(1-\frac{\epsilon_{0}}{\epsilon}\right) \frac{\boldsymbol{b} \boldsymbol{b}}{|\boldsymbol{b}|^{2}}
\end{aligned}
$$

In the final step we used that $\boldsymbol{a}$ and $\boldsymbol{b}$ are orthogonal and span the $x y$-plane, i.e., $\frac{a \boldsymbol{a}}{|\boldsymbol{a}|^{2}}+\frac{\boldsymbol{b} \boldsymbol{b}}{|\boldsymbol{b}|^{2}}=\mathbf{1}$. To first order, the result is then (using $\sin ^{2} \theta=|\boldsymbol{a}|^{2}=|\boldsymbol{b}|^{2}$ )

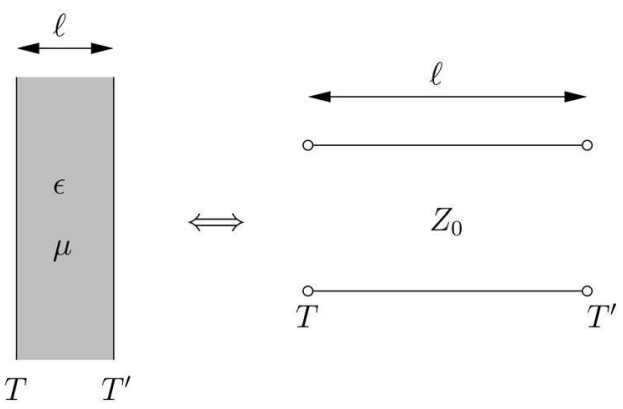

Fig. 1. Transmission line model of an isotropic slab.

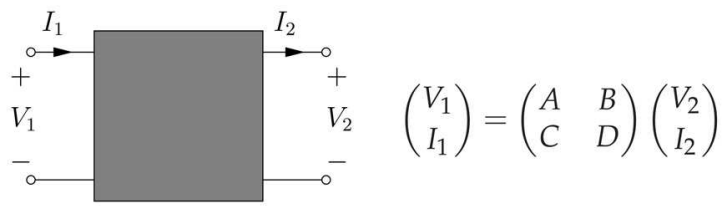

Fig. 2. Definition of ABCD matrix parameters for a general twoport network. 


$$
\left(\begin{array}{cc}
\mathbf{1} & \mathrm{j} \omega\left(\mu-\mu_{0}\right) t \mathbf{1}+\mathrm{j} \omega \mu_{0} t\left(1-\frac{\epsilon_{0}}{\epsilon}\right) \boldsymbol{b} \boldsymbol{b} \\
\mathrm{j} \omega\left(\epsilon-\epsilon_{0}\right) t \mathbf{1}+\mathrm{j} \omega \epsilon_{0} t\left(1-\frac{\mu_{0}}{\mu}\right) \boldsymbol{a} \boldsymbol{a} & \mathbf{1}
\end{array}\right)
$$

Thus, a thin sheet of homogeneous material with permittivity $\epsilon$ and permeability $\mu$ can be modelled to first order as a series impedance $Z$ and shunt admittance $Y$ with the values

$$
\begin{aligned}
& Z=\left\{\begin{array}{lr}
\mathrm{j} \omega\left(\mu-\mu_{0}\right) t & \text { TEpolarization } \\
\mathrm{j} \omega\left(\mu-\mu_{0}\right) t+\mathrm{j} \omega \mu_{0} t\left(1-\epsilon_{0} / \epsilon\right) \sin ^{2} \theta & \text { TMpolarization }
\end{array}\right. \\
& Y= \begin{cases}\mathrm{j} \omega\left(\epsilon-\epsilon_{0}\right) t+\mathrm{j} \omega \epsilon_{0} t\left(1-\mu_{0} / \mu\right) \sin ^{2} \theta & \text { TEpolarization } \\
\mathrm{j} \omega\left(\epsilon-\epsilon_{0}\right) t & \text { TMpolarization }\end{cases}
\end{aligned}
$$

where $t$ is the thickness of the sheet. An important special case is the resistive sheet, where $\epsilon=\epsilon^{\prime}-\mathrm{j} \sigma / \omega$ and $\mu=\mu_{0}$. In the limit $\omega \rightarrow 0$, we then have

$$
Y \rightarrow \sigma t \text { and } Z \rightarrow 0
$$

regardless of polarization. The quantity $1 /(\sigma t)=R_{\mathrm{s}}$ is called the sheet resistance.

To see how sheets with a periodic pattern can be handled, we introduce the electric and magnetic polarizability per unit area $\gamma_{\mathrm{e}} / A$ and $\gamma_{\mathrm{m}} / A$, such that $\epsilon_{0} \gamma_{\mathrm{e}} \cdot \boldsymbol{E}_{0}$ is the static polarization induced in the sheet when subjected to a homogeneous field $E_{0}$. The physical unit of $\gamma_{\mathrm{e}} / A$ and $\gamma_{\mathrm{m}} / A$ is length. The polarizability is in general a dyadic that can be represented as a $3 \times 3$ matrix, with the decomposition

$$
\gamma_{\mathrm{e}}=\gamma_{\mathrm{ett}}+\hat{z} \gamma_{\mathrm{ez}}+\gamma_{\mathrm{et}} \hat{z}+\gamma_{\mathrm{e} z z} \hat{z} \hat{z}
$$

with the corresponding decomposition for the magnetic polarizability. As shown in (Sjöberg, 2009a), the polarizability dyadics can be calculated from the solutions of the following static problems, where $\boldsymbol{E}_{0}$ and $\boldsymbol{H}_{0}$ are given constant vectors,

$$
\begin{gathered}
\nabla \cdot\left[\boldsymbol{\epsilon} \cdot\left(\boldsymbol{E}_{0}-\nabla \varphi_{\mathrm{e}}\right)\right]=0 \\
\nabla \cdot\left[\boldsymbol{\mu} \cdot\left(\boldsymbol{H}_{0}-\nabla \varphi_{\mathrm{m}}\right)\right]=0
\end{gathered}
$$

with periodic boundary conditions in the $x y$-plane and $\nabla \varphi_{\mathrm{e}, \mathrm{m}} \rightarrow 0$ as $z \rightarrow \pm \infty$. In these equations, $\epsilon$ and $\mu$ are the static permittivity and permeability dyadics, which may be anisotropic but are always symmetric and real-valued. The polarizability dyadics are then defined by (where $U$ denotes the unit cell in the $x y$ plane)

$$
\begin{gathered}
\int_{-\infty}^{\infty} \int_{U}\left(\boldsymbol{\epsilon} / \epsilon_{0}-\mathbf{1}\right) \cdot\left(\boldsymbol{E}_{0}-\nabla \varphi_{\mathrm{e}}\right) d S d z=\boldsymbol{\gamma}_{\mathrm{e}} \cdot \boldsymbol{E}_{0} \\
\int_{-\infty}^{\infty} \int_{U}\left(\boldsymbol{\mu} / \mu_{0}-\mathbf{1}\right) \cdot\left(\boldsymbol{H}_{0}-\nabla \varphi_{\mathrm{m}}\right) d S d z=\boldsymbol{\gamma}_{\mathrm{m}} \cdot \boldsymbol{H}_{0}
\end{gathered}
$$


Generalizations of these equations to encompass the possibility of metal inclusions are given in (Sjöberg, 2009a). Using these quantities, the low frequency scattering against a low-pass sheet with periodic structure is (Sjöberg, 2009a)

$$
\begin{array}{r}
\mathbf{t}=\mathbf{1}-\frac{\mathrm{j} k_{0}}{2}\left\{\eta_{0}^{-1} \mathbf{Z}_{0} \cdot\left[\frac{\gamma_{\mathrm{ett}}}{A}+\frac{\gamma_{\mathrm{m} z z}}{A} \boldsymbol{a} \boldsymbol{a}\right]+\left[-\hat{z} \times \frac{\gamma_{\mathrm{mtt}}}{A} \cdot \hat{z} \times+\frac{\gamma_{\mathrm{ez} z}}{A} \boldsymbol{b} \boldsymbol{b}\right] \cdot \mathbf{Z}_{0}^{-1} \eta_{0}\right. \\
\left.+\mathbf{Z}_{0} \cdot\left[\boldsymbol{a} \frac{\gamma_{\mathrm{mz}}}{A}-\frac{\gamma_{\mathrm{et}}}{A} \boldsymbol{a}\right] \cdot \hat{z} \times \mathbf{Z}_{0}^{-1}+\hat{z} \times\left[\boldsymbol{a} \frac{\gamma_{\mathrm{ez}}}{A}-\frac{\gamma_{\mathrm{mt}}}{A} \boldsymbol{a}\right]\right\} \\
\mathbf{r}=-\frac{\mathrm{j} k_{0}}{2}\left\{\eta_{0}^{-1} \mathbf{Z}_{0} \cdot\left[\frac{\gamma_{\mathrm{ett}}}{A}+\frac{\gamma_{\mathrm{m} z z}}{A} \boldsymbol{a} \boldsymbol{a}\right]-\left[-\hat{z} \times \frac{\gamma_{\mathrm{mtt}}}{A} \cdot \hat{z} \times+\frac{\gamma_{\mathrm{e} z z}}{A} \boldsymbol{b} \boldsymbol{b}\right] \cdot \mathbf{Z}_{0}^{-1} \eta_{0}\right. \\
\left.+\mathbf{Z}_{0} \cdot\left[\boldsymbol{a} \frac{\boldsymbol{\gamma}_{\mathrm{mz}}}{A}-\frac{\gamma_{\mathrm{et}}}{A} \boldsymbol{a}\right] \cdot \hat{z} \times \mathbf{Z}_{0}^{-1}-\hat{z} \times\left[\boldsymbol{a} \frac{\gamma_{\mathrm{ez}}}{A}-\frac{\gamma_{\mathrm{mt}}}{A} \boldsymbol{a}\right]\right\}
\end{array}
$$

The cross product with the $z$ direction, $\hat{z} \times$, can be represented as a skew-symmetric matrix which is its own negative inverse. Thus, the expression $-\hat{z} \times \frac{\gamma_{\mathrm{mtt}}}{A} \cdot \hat{z} \times=(\hat{z} \times)^{-1} \cdot \frac{\gamma_{\mathrm{mtt}}}{A} \cdot \hat{z} \times$
is a similarity transform of $\gamma_{\mathrm{mtt}} / A$.

In order to identify the circuit analog of these expressions, we compare with the simple networks in Figure 3 and compute their reflection and transmission coefficients. Assuming $Z_{1}=\mathrm{j} \omega L$ and $Y_{2}=\mathrm{j} \omega C$, all networks in Figure 3 have the same ABCD-matrix to first order in $\omega$,

$$
\left(\begin{array}{ll}
A & B \\
C & D
\end{array}\right)=\left(\begin{array}{cc}
1 & Z_{1} \\
Y_{2} & 1
\end{array}\right) \Rightarrow\left\{\begin{array}{c}
r=\frac{1}{2}\left(Z_{1} / Z_{0}-Y_{2} Z_{0}\right) \\
t=1-\frac{1}{2}\left(Z_{1} / Z_{0}+Y_{2} Z_{0}\right)
\end{array}\right.
$$

where $Z_{0}$ is the characteristic impedance of the surrounding medium. Comparison between the two expressions implies

$$
\begin{aligned}
& \mathbf{Z}_{1} \cdot \mathbf{Z}_{0}^{-1}=\mathrm{j} k_{0}\left\{\left[-\hat{z} \times \frac{\gamma_{\mathrm{mtt}}}{A} \cdot \hat{z} \times+\boldsymbol{b} \boldsymbol{b} \frac{\gamma_{\mathrm{e} z z}}{A}\right] \cdot \mathbf{Z}_{0}^{-1} \eta_{0}+\hat{z} \times\left[\boldsymbol{a} \frac{\gamma_{\mathrm{e} z \mathrm{t}}}{A}-\frac{\gamma_{\mathrm{mtz}}}{A} \boldsymbol{a}\right]\right\} \\
& \mathbf{Z}_{0} \cdot \mathbf{Y}_{2}=\mathrm{j} k_{0}\left\{\eta_{0}^{-1} \mathbf{Z}_{0} \cdot\left[\frac{\gamma_{\mathrm{ett}}}{A}+\boldsymbol{a} \boldsymbol{a} \frac{\gamma_{\mathrm{m} z z}}{A}\right]+\mathbf{Z}_{0} \cdot\left[\boldsymbol{a} \frac{\gamma_{\mathrm{m} z \mathrm{t}}}{A}-\frac{\gamma_{\mathrm{etz}}}{A} \boldsymbol{a}\right] \cdot \hat{\boldsymbol{z}} \times \mathbf{Z}_{0}^{-1}\right\}
\end{aligned}
$$

Using $\mathbf{Z}_{1}=\mathbf{j} \omega \mathbf{L}$ and $\mathbf{Y}_{2}=\mathbf{j} \omega \mathbf{C}$, this implies the sheet series inductance dyadic $\mathbf{L}$ and sheet shunt capacitance dyadic $\mathbf{C}$ is (which generalizes (43) and (44) to anisotropic materials)

$$
\begin{gathered}
\mathbf{L}=\frac{1}{\mathrm{c}_{0}}\left\{\left[-\hat{z} \times \frac{\gamma_{\mathrm{mtt}}}{A} \cdot \hat{z} \times+\frac{\gamma_{\mathrm{ezz}}}{A} \boldsymbol{b} \boldsymbol{b}\right] \eta_{0}+\hat{z} \times\left[\boldsymbol{a} \frac{\gamma_{\mathrm{e} z \mathrm{t}}}{A}-\frac{\gamma_{\mathrm{mtz}}}{A} \boldsymbol{a}\right] \cdot \mathbf{Z}_{0}\right\} \\
\mathbf{C}=\frac{1}{c_{0}}\left\{\eta_{0}^{-1}\left[\frac{\gamma_{\mathrm{ett}}}{A}+\frac{\gamma_{\mathrm{m} z z}}{A} \boldsymbol{a} \boldsymbol{a}\right]+\left[\boldsymbol{a} \frac{\gamma_{\mathrm{m} z \mathrm{t}}}{A}-\frac{\gamma_{\mathrm{etz}}}{A} \boldsymbol{a}\right] \cdot \hat{z} \times \mathbf{Z}_{0}^{-1}\right\}
\end{gathered}
$$




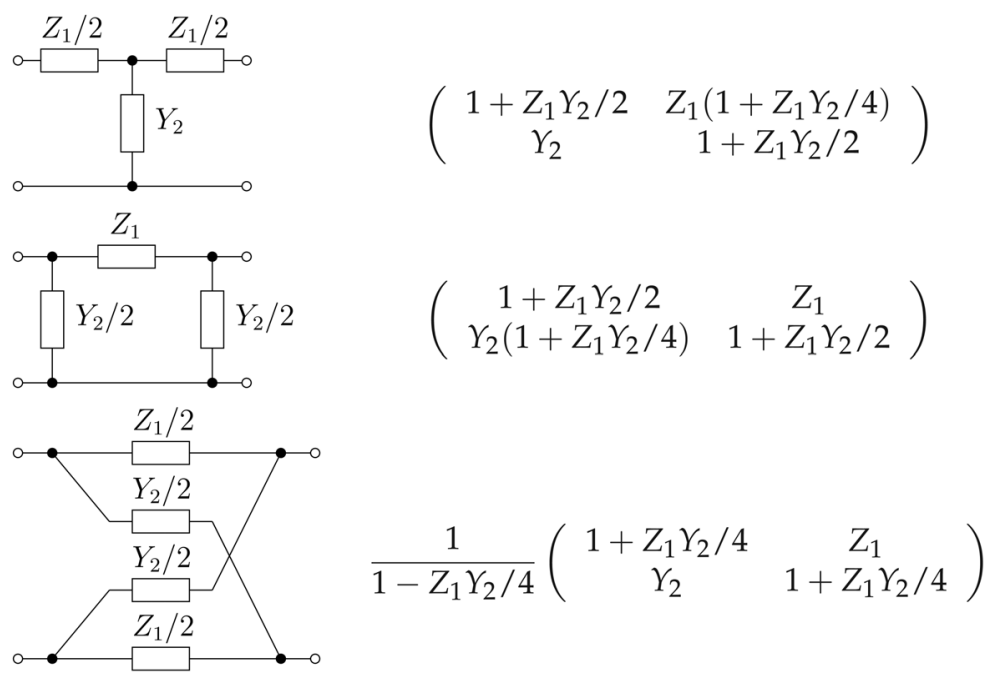

Fig. 3. ABCD-matrices for symmetric $T, \Pi$, and trellis net.

These dyadics are represented by diagonal matrices if there is no coupling between TE and TM modes. For normal incidence on an isotropic slab with thickness $t$, the parameters take on the simple scalar values

$$
L=\left(\mu-\mu_{0}\right) t \text { and } \quad C=\left(\epsilon-\epsilon_{0}\right) t
$$

Note that the circuit parameters defined in this section correspond to a low frequency expansion, where the sheet is considered thin in terms of wavelength. For higher frequencies, the method presented in Section 5 can be used.

\section{Physical limitations}

Circuit analogs appear in a very natural way when considering physical limitations of scattering against stratified structures. The methodology dates back to classical work on optimum matching (Fano, 1950), using clever integration paths in the complex plane for functions representing linear, causal, passive systems. In physics, the corresponding relations are known as sum rules, connecting an integral over all frequencies of some quantity to the static value of another (Nussenzveig, 1972). Often, the sum rules are derived from relations similar to the Kramers-Kronig's relations (de L. Kronig, 1926; Kramers, 1927). In this section, we only give the final results of other authors' work, and refer to the original papers for more in depth discussions.

The first paper to discuss physical limitations on scattering from planar structures was by (Rozanov, 2000). He derived the following limitation on the reflection coefficient $R$ from any metal-backed planar structure (where $\lambda=c_{0} / f$ is the wavelength in vacuum):

$$
\int_{0}^{\infty} \ln \left(\frac{1}{|r(\lambda)|}\right) d \lambda \leq 2 \pi^{2} \sum_{i} \mu_{\mathrm{s}, i} d_{i}=2 \pi^{2} \frac{L}{\mu_{0}}
$$


Here, we identify the inductance as $L=\mu d$ instead of $\left(\mu-\mu_{0}\right) d$, since the reference plane of the reflection is at the top of the structure and not at the ground plane. The expression (59) demonstrates that the bandwidth over which the amplitude of the reflection coefficient is less than unity, is bounded above by the static permeability of the structure, which can be interpreted as the low frequency series inductance. The interesting part of this physical limitation is that it is valid for any realization of the structure, and provides a useful upper bound for absorbers. This is seen from the fact that the integral is bounded below by $\left(\lambda_{2}-\lambda_{1}\right)$ $\ln \left(1 / r_{0}\right)$, where $r_{0}$ is the largest reflection level in the band $\left[\lambda_{1}, \lambda_{2}\right]$. Using the relative bandwidth $B=\left(\lambda_{2}-\lambda_{1}\right) / \lambda_{0}$, where the center wavelength is $\lambda_{0}=\left(\lambda_{1}+\lambda_{2}\right) / 2$, we find

$$
B \ln \left(\frac{1}{r_{0}}\right) \leq 2 \pi^{2} \frac{\sum \mu_{\mathrm{s}, i} d_{i}}{\lambda_{0}}=2 \pi^{2} \frac{L}{\lambda_{0} \mu_{0}}
$$

Thus, the product of bandwidth and reflection level in logarithmic scale is bounded above by a factor proportional to the low frequency series inductance of the structure.

A similar bound was found by (Brewitt-Taylor, 2007) for the realization of artificial magnetic conductors, by studying the factor $P=(r-1) / 2$. Magnetic conductors are attractive in antenna design problems, and are characterized by a reflection coefficient $r \approx+1$, meaning $P$ becomes small in the band of interest. The bound is

$$
\int_{0}^{\infty} \ln \left(\frac{1}{|P(\lambda)|}\right) d \lambda \leq \pi^{2} \sum_{i} \mu_{\mathrm{s}, i} d_{i}=\pi^{2} \frac{L}{\mu_{0}}
$$

with similar interpretation as Rozanov's result and corresponding bandwidth bound. Our final example is of the transmission through a periodic low-pass screen (Gustafsson et al., 2009), where the following bound for a non-magnetic structure was derived (where $t$ is the transmission coefficient)

$$
\int_{0}^{\infty} \ln \frac{1}{|t(\lambda)|} d \lambda \leq \pi^{2} \frac{E_{0} \cdot \gamma_{\mathrm{ett}} \cdot E_{0}}{2 A\left|E_{0}\right|^{2}}=\frac{\pi^{2}}{2} \frac{C}{\epsilon_{0}}
$$

The factor $\gamma_{\text {ett }} / A$ is the capacitance dyadic in (57) for normal incidence, and similar physical bounds can be derived for antennas, materials and general scatterers (Sohl et al., 2007a; Gustafsson et al., 2007; Sohl et al., 2007a;b; 2008; Sohl \& Gustafsson, 2008). When considering the physical limitations, it is noteworthy that the circuit parameters (or rather, the polarizability dyadics) can be bounded using variational principles as discussed in (Sjöberg, 2009b). These typically state that the polarizability of a given structure cannot decrease if we add more material; in particular, the electric polarizability of any body is always less than (or at most equal to) the polarizability of a circumscribed metallic body. If the metallic body has a simple shape (such as a sphere), its polarizability can be computed analytically, and hence a useful approximation of the polarizability of the original body is provided.

\section{Computation of circuit analogs in the general case}

So far, we have only demonstrated how to compute circuit analogs in the low frequency limit. Indeed, this is the primary region where we can give firm definitions and physical 


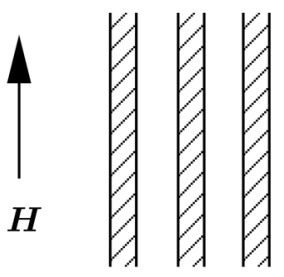

Front view

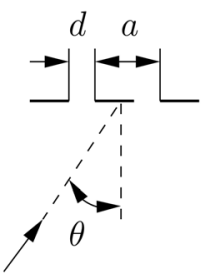

Top view

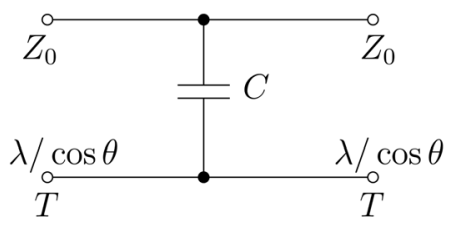

Equivalent circuit

Fig. 4. Geometry and equivalent circuit for capacitive strips (TM polarization).

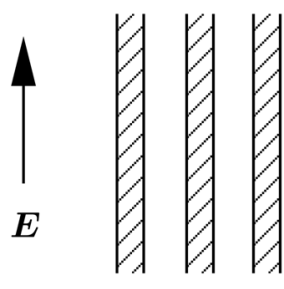

Front view

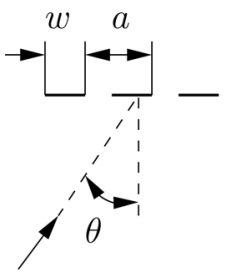

Top view

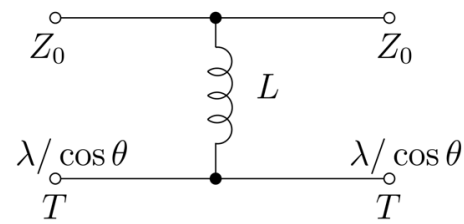

Equivalent circuit

Fig. 5. Geometry and equivalent circuit for inductive strips (TE polarization).

interpretations of the circuit analogs, but analogs are still valuable as a modeling tool even for higher frequencies, in particular for structures of subwavelength size. The general procedure is the same as previously employed: the circuit analogs are computed to provide the same scattering characteristics as the full structure.

Many analytical expressions have been derived throughout the years in the microwave literature, in particular associated with the development of radar technology during the Second World War. Many of these results are collected in references such as (Collin, 1991; Marcuvitz, 1951; Schwinger \& Saxon, 1968). The most explored geometry is that of metallic strips, see Figures 4 and 5. Depending on the polarization of the incident wave, the strips behave dominantly capacitive or inductive. Provided that the width of the strips and the distance between them can be considered small in terms of wavelengths, the circuit parameters in Figures 4 and 5 can be estimated as follows (Marcuvitz, 1951, pp. 280 and 284)

$$
C \approx \frac{4 a \epsilon_{0}}{\pi} \ln \frac{2 a}{\pi d} \text { and } L \approx \frac{a \mu_{0}}{2 \pi} \ln \frac{2 a}{\pi w}
$$

Note that the inductance $L$ is now a shunt inductance, in contrast to the series inductance obtained by transmission through a thin slab. We can immediately interpret these results in order to gain some design intuition:

- $\quad$ To make the capacitance $C$ large, the ratio $d / a$ should be small, i.e., the gap between the strips should be small.

- To make the inductance $L$ large, the ratio $w / a$ should be small, i.e., the width of the strips should be small. 


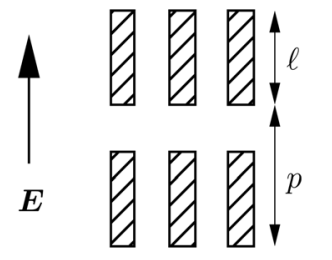

Front view

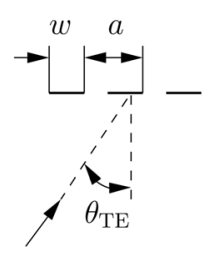

Top view

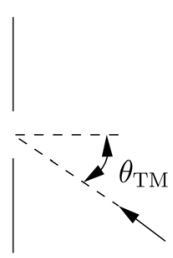

Side view

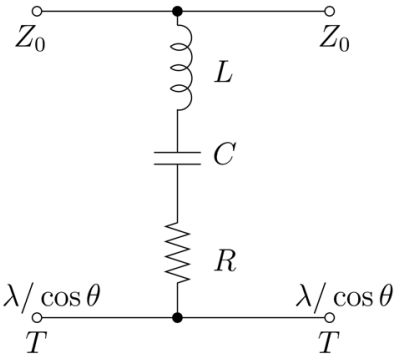

Equivalent circuit

Fig. 6. Finite dipole. Observe that the incidence plane changes depending on whether we study TE or TM polarization, since the dipoles would be practically invisible for other cases.

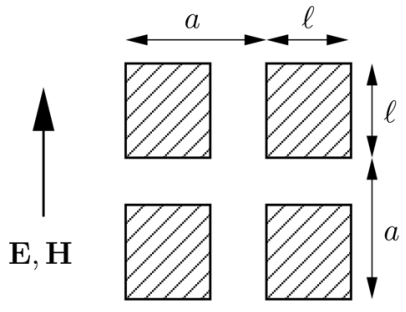

Front view

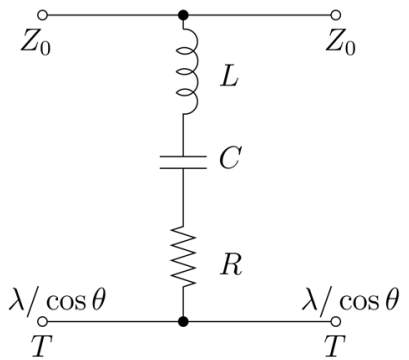

Equivalent circuit

Fig. 7. A pattern of square patches. Compared to the finite dipoles in Figure 6, this structure is relatively independent of polarization, due to its higher symmetry.

When the strips are not infinite, a more sophisticated modeling must be made. For an array of finite strips as in Figure 6, we need to incorporate more elements in order to reflect the possibility of resonance, when the dipoles become approximately half a wavelength. If the metal strips are lossy, we also need to include a resistance in the circuit analog. It is not trivial to compute the exact circuit parameters in this case, and in practice a numerical approach is necessary. Also, the thin finite dipoles are polarization sensitive, being essentially invisible to electric fields orthogonal to their longest extension. By using square patches as in Figure 7, a higher symmetry is achieved and thereby less polarization sensitivity. On the other hand, since the patch is wide, its inductance tends to be smaller than the corresponding dipole.

Many practical structures can be considered to be thin and non-magnetic. In this case, the simple model in Figure 8 can be used, where the sheet is considered as a single shunt lumped element, possibly with a complicated frequency dependence. To compute the relevant circuit analog for an arbitrary such sheet, we can make a full wave simulation to compute scattering parameters such as the reflection coefficient $r(\omega)$. From this given data we can turn to the circuit model in Figure 8 to find the reflection coefficient

$$
r=\frac{Y_{0}-\left(Y+Y_{0}\right)}{Y+\left(Y+Y_{0}\right)}=\frac{1-\left(Y+Y_{0}\right) / Y_{0}}{1+\left(Y+Y_{0}\right) / Y_{0}} \Rightarrow \frac{Y+Y_{0}}{Y_{0}}=\frac{1-r}{1+r}
$$



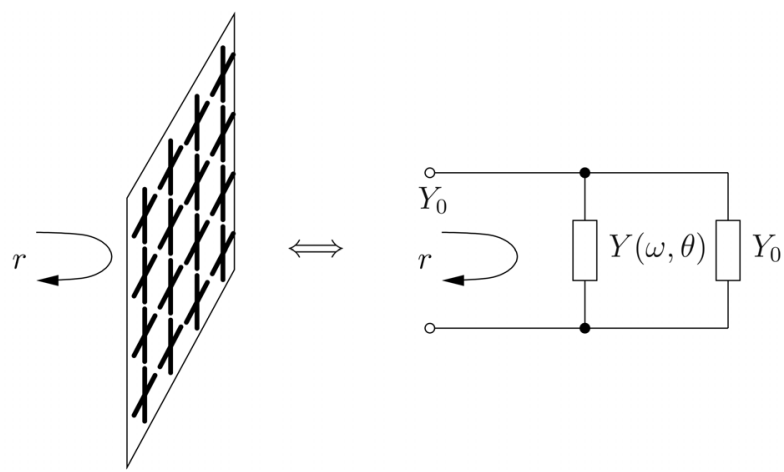

Fig. 8. Equivalent circuit model for reflection against a nonmagnetic sheet backed by free space. The shunt admittance $Y$ is usually a function of frequency and angle of incidence, as well as polarization.

Thus, we can compute a numerical value for the normalized surface admittance $\left(Y+Y_{0}\right) / Y_{0}$ for each frequency $\omega$. The next step is to match this data with a rational approximant $\left(Y+Y_{0}\right) / Y_{0}=p(s) / q(s)$, where $p$ and $q$ are polynomials in $s=\mathrm{j} \omega$. In order for the identified model $p(s) / q(s)$ to correspond to a realizable circuit, it is necessary that all zeros of $q(s)$ have negative real part. This can be achieved by using matlab's routine invfreqs.

To compute the numerical reflection coefficients $r(\omega)$, it is necessary to reduce the problem to a unit cell with periodic boundary conditions in the $x y$-plane, and plane wave ports in the $z$ direction. These requisites are commonly available in most advanced simulation programs today.

\section{Applications}

The usefulness of circuit analogs resides in the fact that they are a compact representation of the scattering properties of a given structure. In particular, in design problems where many potential designs need to be evaluated, circuit analogs provide a convenient means of producing concept designs, which must later be refined and verified using full-wave simulations.

One design methodology, is to choose a general geometry for the sheets such as the finite dipoles in Figure 6, and then build a database of corresponding circuit parameters for different geometry parameters. The desired electromagnetic function, for instance absorption over a certain bandwidth, is then first treated as a circuit design problem. Once a circuit design is found, typically consisting of segments of transmission lines loaded by lumped elements, the realization is found by lookup in the previously calculated tables. This methodology works well when the individual sheets are about a quarter wavelength from each other.

The method breaks down when the sheets become closely spaced, say within a tenth of a wavelength. There is then substantial coupling between the sheets through the otherwise evanescent fields, so that the previous circuit models do not apply anymore. However, new circuit models can be applied to clusters of sheets, although the number of geometry parameters may become so large that it becomes difficult to build a reasonable database. 


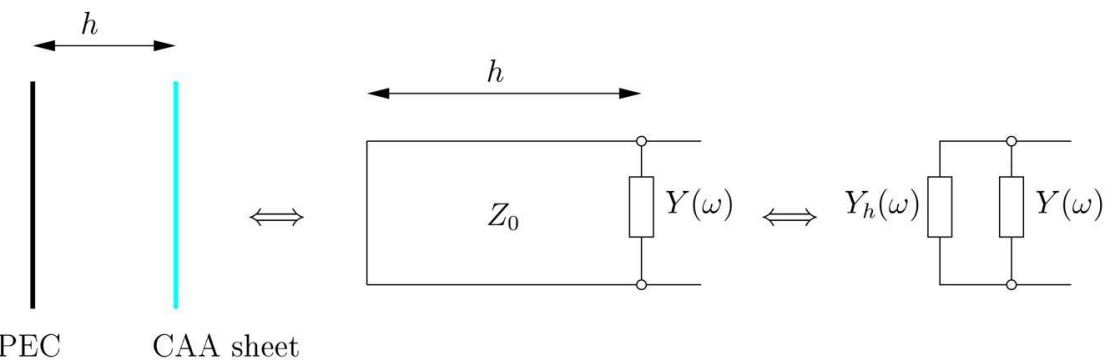

Fig. 9. Typical situation for a Circuit Analog Absorber (CAA) sheet. A CAA sheet is situated at height $h$ above a metallic ground plane, and is modeled as a lumped shunt element in a corresponding transmission line. The shorted transmission line can be transformed to a lumped load $Y_{h}(\omega)$, shunting the sheet admittance $Y(\omega)$.

In (Sjöberg, 2008), this design methodology is demonstrated for electromagnetic absorbers as in Figure 9. A typical absorber structure is the Salisbury screen, where a resistive sheet of sheet resistance $R_{\mathrm{s}}=1 /(\sigma t)=Z_{0}=\sqrt{\mu_{0} / \epsilon_{0}}=377 \Omega$ is placed at height $h$ above a ground plane. The short circuit of the transmission line provided by the ground plane is transformed to the location of the sheet as (Pozar, 2005, p. 60)

$$
Y_{h}(\omega)=-\mathrm{j} Y_{0} \cot \left(k_{0} h \cos \theta\right)
$$

where $k_{0}=\omega / c_{0}$ is the free space wave number, $\theta$ is the angle of incidence, and $Y_{0}$ is the characteristic admittance of the transmission line (the inverse of $Z$ in (33)). For normal incidence, it is seen that $Y_{h}\left(\omega_{0}\right)=0$ for $k_{0} h=\pi / 2$, implying the load impedance as seen by the incident wave is $Z_{\mathrm{L}}=1 /\left(1 / R_{\mathrm{S}}+Y_{h}\right)=R_{\mathrm{s}}=Z_{0}$ at this frequency, providing zero reflection since the transmission line is then matched. The condition $k_{0} h=\pi / 2$ can be interpreted as $h$ being equal to a quarter wavelength at the design frequency $\omega_{0}$. The relative bandwidth is about $25 \%$ for $-20 \mathrm{~dB}$ reflectivity level (Knott et al., 2004, p. 316) corresponding to the bandwidth of the requirement $\cot \left(k_{0} h\right)=0$. However, the bound (60) given by (Rozanov, 2000) suggests that the upper bound on the bandwidth at $-20 \mathrm{~dB}$ well exceeds $100 \%$, suggesting the design can be improved.

One improvement is to include a pattern in the resistive sheet, using the additional reactive elements to increase the bandwidth. Results of this method are depicted in Figures 10 and 11. It is seen that the design in Figure 10, based only on the pure circuit design and circuit analog representation of the sheet (details can be found in (Sjöberg, 2008)), produces a structure whose full wave characteristics are not optimal. A slight tuning of the geometry results in Figure 11, which has a better full wave result. The full wave simulations were made with the program PB-FDTD, which uses finite differences in the time-domain with periodic boundary conditions (Holter \& Steyskal, 1999). We note that neither of these designs is close to the optimal limit by Rozanov. More advanced designs based on capacitive squares close to the ground plane can come close to the optimal limit (Kazem Zadeh \& Karlsson, 2009). A design procedure taking oblique incidence into account can be found in (Munk et al., 2007).

Another design case which is made easier using circuit analogs is the construction of a linear-to-circular polarizer using meander lines, see (Young et al., 1973; Terret et al., 1984) and (Munk, 2003, pp. 306-326). In order to create circular polarization from linear 

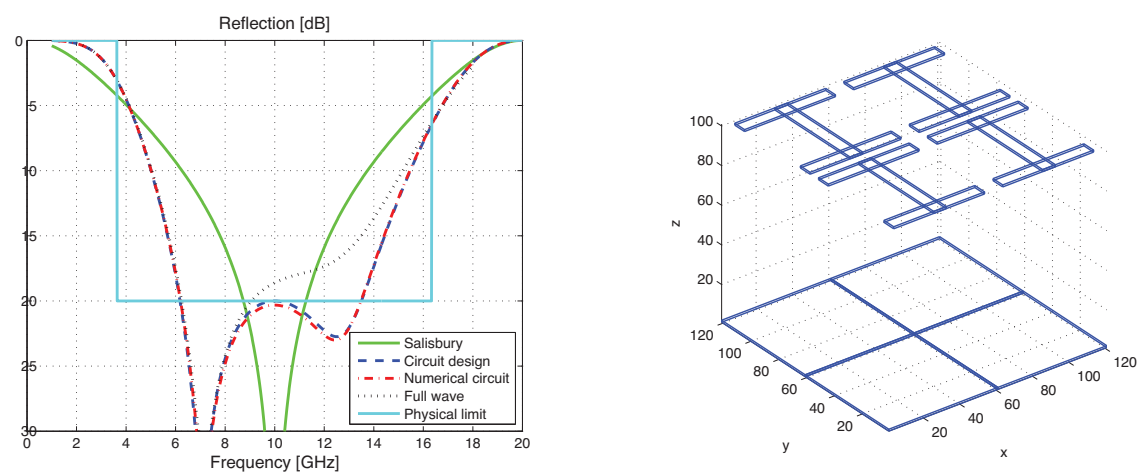

Fig. 10. Comparison between a Salisbury screen and a circuit analog absorber (total height is $7.5 \mathrm{~mm}$, normal incidence). The "ideal" circuit parameters computed from a circuit design are $R=308 \Omega, C=80.4 \mathrm{fF}$, and $L=3.15 \mathrm{nH}$. These parameters are approximately achieved by the geometry depicted on the right, where the optimization procedure described after equation (64) predicts $R=311 \Omega, C=80.4 \mathrm{fF}$, and $L=3.16 \mathrm{nH}$. These parameters are used in a circuit model, and the full wave results are also displayed. The physical limit (60) is represented by the square box, demonstrating that there is room for improvement on this design.
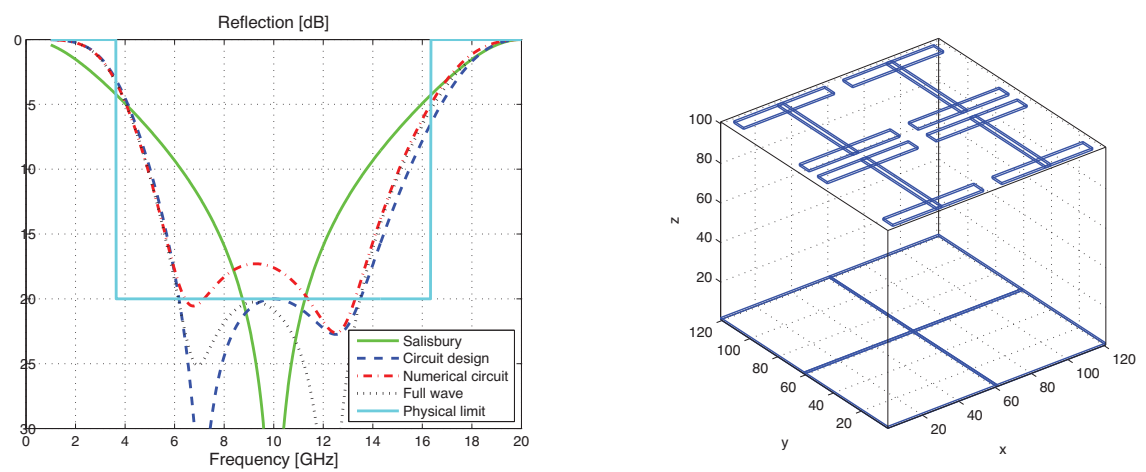

Fig. 11. A version of the CAA pattern in Figure 10 with more slender center part, and lower resistance per square. This provides higher inductance and therefore lower resonance frequency than the structure in Figure 10. The circuit parameters are $R=319 \Omega, C=79.1 \mathrm{fF}$, and $L=4.35 \mathrm{nH}$.

polarization, it is common to use a structure which delays the $x$-component $90^{\circ}$ relative the $y$-component; by sending in the linear polarization with equal strength in $x$ and $y$, the result is a circularly polarized wave. One idea to create this delay is to use the behavior of the metal strips in Figures 4 and 5, which are dominantly inductive for electric fields polarized along them, and dominantly capacitive for electric fields polarized orthogonally to them. In order to increase the reactive properties the lines are meandered, and several sheets are layered in order to achieve the total phase shift necessary. As is explained in detail in (Munk, 2003, pp. 306-326), it is necessary to adjust the outmost layers in order to reduce the 
reflection from the structure, leading to the final design geometry in Figure 12. It is seen in Figure 13 that the results are quite broad band. In the real application, further dielectric sheets need to be added to the design for mechanical rigidity.

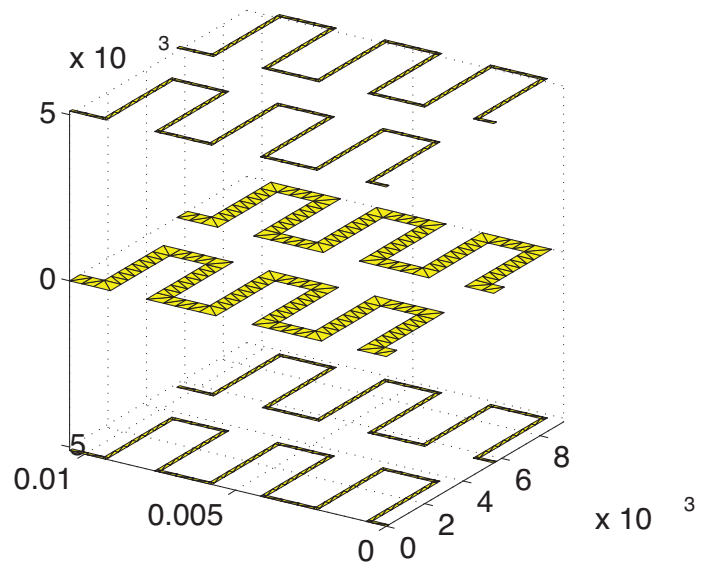

Fig. 12. Geometry of the layered meander structure, making up a linear-to-circular polarizer.
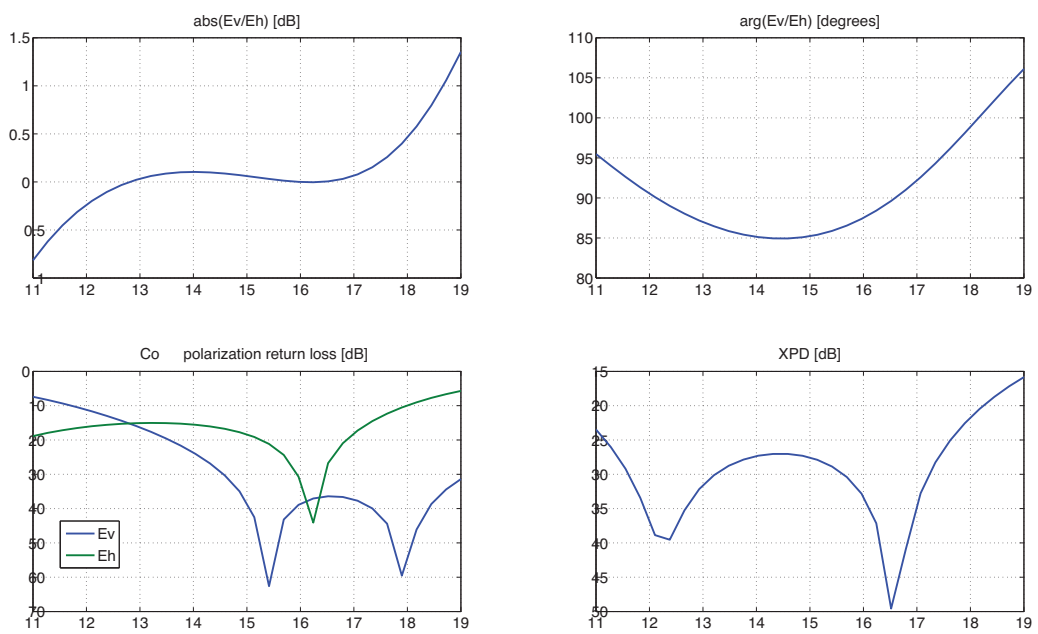

Fig. 13. Results for the layered meander structure (the $x$-axes in all subfigures are frequency in $\mathrm{GHz}$ ). Upper left: the ratio between the vertical and horizontal polarization of the transmitted field. Upper right: the phase difference between the polarizations. Lower left: co-polarization reflection. Lower right: cross-polarization discrimination. 


\section{Conclusions}

We have shown how relatively complex wave propagation problems can be efficiently modeled and designed using circuit analogs. Propagation of plane waves in any bianisotropic material can be modeled as propagation of voltage and current in two transmission lines, one for each polarization. The wave number and characteristic impedance for these transmission lines are determined from the algebraic eigenvalue problem (28). For isotropic media, there is no coupling between the TE and TM polarization, but in general we must allow for this coupling by using a dyadic transverse impedance.

Scatterers such as thin sheets, with or without a periodic pattern, may be modeled as lumped elements. If the sheets are non-magnetic, they can be reduced to a single shunt element. The circuit parameters can be determined in the static limit using the electric and magnetic polarizability per unit area. For higher frequencies, more advanced calculations taking the finite wavelength into account must be applied, for instance numerically.

The strength of the simple circuit analogs lies in their use in concept design, where a large number of possible realizations of a specified function must be evaluated. Using the simple models, reliable concept designs can be provided which are subsequently subjected to a more detailed (and costly) analysis and refined, to provide the final design.

\section{References}

Brewitt-Taylor, C. R. (2007). Limitation on the bandwidth of artificial perfect magnetic conductor surfaces, Microwaves, Antennas \& Propagation, IET 1(1): 255-260.

Caloz, C. \& Itoh, T. (2004). Transmission line approach of left-handed (LH) materials and microstrip implementation of an artificial LH transmission line, IEEE Trans. Antennas Propagat. 52(5): 1159-1166.

Collin, R. E. (1991). Field Theory of Guided Waves, second edn, IEEE Press, New York.

Collin, R. E. (1992). Foundations for Microwave Engineering, second edn, McGraw-Hill, New York.

de L. Kronig, R. (1926). On the theory of dispersion of X-rays, J. Opt. Soc. Am. 12(6): 547-557.

Eleftheriades, G. V., Iyer, A. K. \& Kremer, P. C. (2002). Planar negative refractive index media using periodically loaded L-C loaded transmission lines, IEEE Trans. Microwave Theory Tech. 50(12): 2702-2712.

Fano, R. M. (1950). Theoretical limitations on the broadband matching of arbitrary impedances, Journal of the Franklin Institute 249(1,2): 57-83 and 139-154.

Gustafsson, M., Sohl, C. \& Kristensson, G. (2007). Physical limitations on antennas of arbitrary shape, Proc. R. Soc. A 463: 2589-2607.

Gustafsson, M., Sohl, C., Larsson, C. \& Sjöberg, D. (2009). Physical bounds on the allspectrum transmission through periodic arrays, EPL Europhysics Letters 87(3): 34002 (6pp). URL: $h$ ttp://stacks.iop.org/0295-5075/87/34002

Holter, H. \& Steyskal, H. (1999). Infinite phased-array analysis using FDTD periodic boundary conditions - pulse scanning in oblique directions, IEEE Trans. Antennas Propagat. 47(10): 1508-1514.

Kazem Zadeh, A. \& Karlsson, A. (2009). Capacitive circuit method for fast and efficient design of wideband radar absorbers, IEEE Trans. Antennas Propagat. 57(8): 23072314. 
Knott, E. F., Shaeffer, J. F. \& Tuley, M. T. (2004). Radar Cross Section, SciTech Publishing Inc., 5601 N. Hawthorne Way, Raleigh, NC 27613.

Kramers, M. H. A. (1927). La diffusion de la lumière par les atomes, Atti. Congr. Int. Fis. Como 2: 545-557.

Marcuvitz, N. (1951). Waveguide Handbook, McGraw-Hill, New York.

Munk, B. (2000). Frequency Selective Surfaces: Theory and Design, John Wiley \& Sons, New York.

Munk, B. (2003). Finite Antenna Arrays and FSS, John Wiley \& Sons, New York.

Munk, B. A., Munk, P. \& Pryor, J. (2007). On designing Jaumann and circuit analog absorbers (CA absorbers) for oblique angle of incidence, IEEE Trans. Antennas Propagat. 55(1): 186-193.

Nussenzveig, H. M. (1972). Causality and dispersion relations, Academic Press, London.

Poulsen, S. (2006). Stealth radomes, PhD thesis, Lund University, Department of Electroscience, Lund University, P.O. Box 118, S-221 00 Lund, Sweden.

Pozar, D. M. (2005). Microwave Engineering, third edn, John Wiley \& Sons, New York.

Rozanov, K. N. (2000). Ultimate thickness to bandwidth ratio of radar absorbers, IEEE Trans. Antennas Propagat. 48(8): 1230-1234.

Saleh, B. E. A. \& Teich, M. C. (1991). Fundamentals of Photonics, John Wiley \& Sons, New York.

Schwinger, J. \& Saxon, D. S. (1968). Discontinuities in Waveguides, Gordon and Breach Science Publishers, London.

Sjöberg, D. (2008). Analysis of wave propagation in stratified structures using circuit analogs, with application to electromagnetic absorbers, Eur. J. Phys. 29: 721-734.

Sjöberg, D. (2009a). Low frequency scattering by passive periodic structures for oblique incidence: low pass case, J. Phys. A: Math. Theor. 42: 385402.

Sjöberg, D. (2009b). Variational principles for the static electric and magnetic polarizabilities of anisotropic media with perfect electric conductor inclusions, J. Phys. A: Math. Theor. 42: 335403.

Sohl, C. \& Gustafsson, M. (2008). A priori estimates on the partial realized gain of UltraWideband (UWB) antennas, Quart. J. Mech. Appl. Math. 61(3): 415-430.

Sohl, C., Gustafsson, M. \& Kristensson, G. (2007a). Physical limitations on broadband scattering by heterogeneous obstacles, J. Phys. A: Math. Theor. 40: 11165-11182.

Sohl, C., Gustafsson, M. \& Kristensson, G. (2007b). Physical limitations on metamaterials: Restrictions on scattering and absorption over a frequency interval, J. Phys. D: Applied Phys. 40: 7146-7151.

Sohl, C., Larsson, C., Gustafsson, M. \& Kristensson, G. (2008). A scattering and absorption identity for metamaterials: experimental results and comparison with theory, $J$. Appl. Phys. 103(5): 054906.

Terret, C., Levrel, J. R. \& Mahdjoubi, K. (1984). Susceptance computation of a meander-line polarizer layer, IEEE Trans. Antennas Propagat. 32(9): 1007-1011.

Young, L., Robinson, L. A. \& Hacking, C. A. (1973). Meander-line polarizer, IEEE Trans. Antennas Propagat. 21(3): 376-378. 


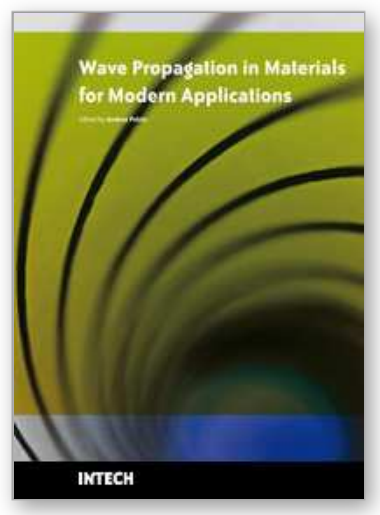

\title{
Wave Propagation in Materials for Modern Applications
}

Edited by Andrey Petrin

ISBN 978-953-7619-65-7

Hard cover, 526 pages

Publisher InTech

Published online 01, January, 2010

Published in print edition January, 2010

In the recent decades, there has been a growing interest in micro- and nanotechnology. The advances in nanotechnology give rise to new applications and new types of materials with unique electromagnetic and mechanical properties. This book is devoted to the modern methods in electrodynamics and acoustics, which have been developed to describe wave propagation in these modern materials and nanodevices. The book consists of original works of leading scientists in the field of wave propagation who produced new theoretical and experimental methods in the research field and obtained new and important results. The first part of the book consists of chapters with general mathematical methods and approaches to the problem of wave propagation. A special attention is attracted to the advanced numerical methods fruitfully applied in the field of wave propagation. The second part of the book is devoted to the problems of wave propagation in newly developed metamaterials, micro- and nanostructures and porous media. In this part the interested reader will find important and fundamental results on electromagnetic wave propagation in media with negative refraction index and electromagnetic imaging in devices based on the materials. The third part of the book is devoted to the problems of wave propagation in elastic and piezoelectric media. In the fourth part, the works on the problems of wave propagation in plasma are collected. The fifth, sixth and seventh parts are devoted to the problems of wave propagation in media with chemical reactions, in nonlinear and disperse media, respectively. And finally, in the eighth part of the book some experimental methods in wave propagations are considered. It is necessary to emphasize that this book is not a textbook. It is important that the results combined in it are taken "from the desks of researchers". Therefore, I am sure that in this book the interested and actively working readers (scientists, engineers and students) will find many interesting results and new ideas.

\section{How to reference}

In order to correctly reference this scholarly work, feel free to copy and paste the following:

Daniel Sjöberg (2010). Circuit Analogs for Wave Propagation in Stratified Structures, Wave Propagation in Materials for Modern Applications, Andrey Petrin (Ed.), ISBN: 978-953-7619-65-7, InTech, Available from: http://www.intechopen.com/books/wave-propagation-in-materials-for-modern-applications/circuit-analogs-forwave-propagation-in-stratified-structures

\section{INTECH}

open science | open minds

\author{
InTech Europe \\ University Campus STeP Ri
}

\section{InTech China}

Unit 405, Office Block, Hotel Equatorial Shanghai 
Slavka Krautzeka 83/A

51000 Rijeka, Croatia

Phone: +385 (51) 770447

Fax: +385 (51) 686166

www.intechopen.com
No.65, Yan An Road (West), Shanghai, 200040, China 中国上海市延安西路65号上海国际贵都大饭店办公楼405单元 Phone: +86-21-62489820

Fax: $+86-21-62489821$ 
(C) 2010 The Author(s). Licensee IntechOpen. This chapter is distributed under the terms of the Creative Commons Attribution-NonCommercialShareAlike-3.0 License, which permits use, distribution and reproduction for non-commercial purposes, provided the original is properly cited and derivative works building on this content are distributed under the same license. 